\title{
Omicron Variant (B.1.1.529) of SARS-CoV-2, A Worldwide Public Health Emergency!
}

\author{
Selia Chowdhury, Mehedi Hasan Bappy, Samia Chowdhury, Md. Shahraj Chowdhury, and Nurjahan \\ Shipa Chowdhury
}

\section{ABSTRACT}

B.1.1.529 is the newest form of SARS- CoV-2. It was initially reported to the World Health Organization (WHO) on November 24, 2021, by South Africa. WHO recognized it as a variant of concern on November 26, 2021, and named it "Omicron." The Omicron variant features an exceptionally large number of mutations, many of which are unique, and a substantial number of which impair the spike protein targeted by most COVID-19 vaccines at the time of its discovery. Despite initial studies showing that the variant caused less serious disease than prior strains, this amount of miscellany has raised worries about its transmissibility, immune system evasion, and vaccine resistance. Omicron is suspected to be much more infectious than previous variants of concerns, spreading around $\mathbf{7 0}$ times faster in the bronchi, but it is less able to penetrate deep lung tissue, which may explain why there is a significant reduction in the risk of severe disease requiring hospitalization. Nonetheless, because of the virus's fast rate of transmission and capacity to resist both two-dose vaccination and the immune system, the overall number of patients requiring medical care at present remains a major worry. In this article, the current state of the art for Omicron variant has been discussed to provide a strong framework for future research. The evolution, mutation, epidemiology, infectivity, vaccine breakthrough, and antibody resistance of the Omicron variant are investigated and discussed.

Keywords: COVID-19, epidemiology, mutation, omicron, SARS-CoV-2, vaccine efficacy.

\section{INTRODUCTION}

SARS-CoV-2 is changing its genetic structure as a result of mutations in order to enhance its ability to evade the immune system, making vaccine development and eventual escape from the pandemic increasingly more difficult [1]. So far, several SARS-CoV-2 variants have been found; some have been labeled as variants of concern (VOCs), while others have been labeled as variants of interest (VOIs) due to potential public health implications. Increased transmissibility decreased neutralization by natural or vaccine-induced antibodies, the ability to elude detection, and a reduction in vaccination or treatment efficacy have all been linked to VOCs [2], [3].

The discovery of the most mutated variant of coronavirus has aroused worry throughout the world. On November 26, 2021, the World Health Organization (WHO) identified this novel variant as a VOC and termed it Omicron (B.1.1.529). After Alpha, Beta, Gamma, and Delta, it is the fifth variant of concern. Australia, Italy, Germany, the Netherlands, Israel, Hong Kong, the United Kingdom, Botswana, and Belgium have all detected Omicron variant among their populations since the first identification in South Africa [4]. This variant has undergone 50 mutations and has over 30 alterations in its spike protein [5]. To combat a coronavirus infection, the immune system produces antibodies that mostly target the spike protein. Because this variant has many mutations that may resist neutralization, researchers are still unsure about the efficiency of vaccinations against it [6]. The most concerning aspect is that Omicron differs greatly from the first coronavirus discovered in Wuhan, China. WHO issued a warning to nations about this novel variation because of its increased transmissibility, faster transmission, and negative impact on the COVID-19 epidemiology. This novel variant Omicron, according to experts, contains an extremely unique constellation of mutations. As a result, the severely muted Omicron variant sparked fears that its surge may escape antibodies developed by past coronavirus infection or immunization [7].

\section{EVOLUTION AND EPIDEMIOLOGY}

Omicron variant has been reported to drive rapid epidemic growth since its identification in November 2021. This variant was first detected on November 8, 2021, in South Africa and in the next two weeks more than 70 percent of sequenced samples from Gauteng province in South Africa was Omicron. By November 25, 2021, it has been identified 
in five different countries and on November 26, 2021, WHO declared Omicron as a variant of concern [8]. United Kingdom reported its first two cases of Omicron on November 27. On December 1, United States identified its first Omicron case along with South Korea, Norway, Saudi Arabia, Ghana, and Nigeria. By December 11, USA reported almost $7 \%$ of the COVID cases to be due to Omicron variant. This rate quickly surged to about $23 \%$ and $60 \%$, respectively, in the next two weeks [9]. Currently, the variant has been found in all the U.S. states and has become the dominant variant in a mere three weeks' time since its first identification in the USA.

$\begin{array}{ccc}\text { November 8, 2021 } & \text { November 24, 2021 } & \text { November 26, 2021 } \\ \text { First confirmed Omicron } & \text { South Africa reported } & \text { WHO designated } \\ \text { sample (in South Africa) } & \text { first Omicron case to } & \text { Omicron as the VOC } \\ & \text { WHO }\end{array}$

$\begin{array}{cc}\text { November 30, 2021 } & \text { December 1, 2021 } \\ \text { USA designated } & \text { USA announced first } \\ \text { Omicron as VOC } & \text { Omicron case }\end{array}$

December 20, 2021

$\mathrm{CDC}$ estimates that Omicron accounts for $73 \%$ of COVID-19 cases in the

U.S. It has been identified in at least 30 states.

December 29, 2021

CDC revised Omicron estimate, comprising $73 \%$ to $59 \%$ of all Covid cases in the U.S. Omicron has spread to all 50 US states.

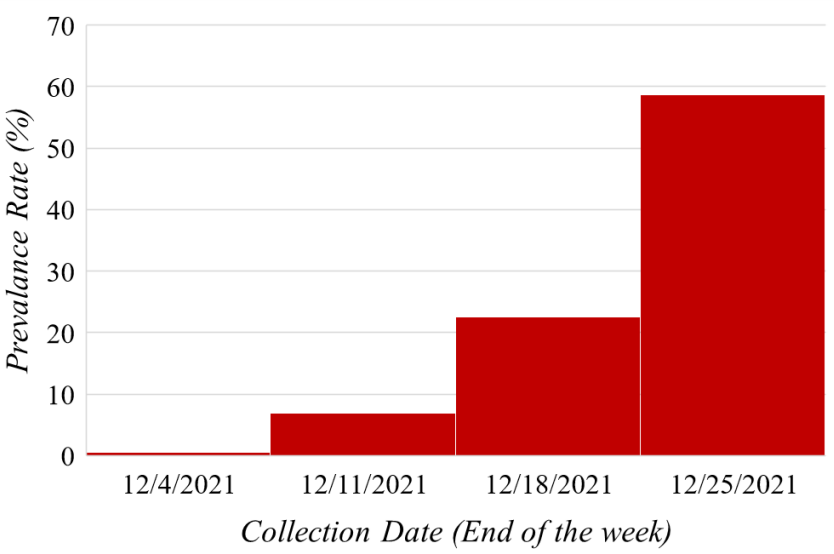

Fig. 1. Key timeline of the evolution of Omicron variant (top) and the prevalence rate in the USA (bottom).

According to phylogenetic analysis, the Omicron variant diverged in late September or early October 2021 [10]. Omicron has been observed frequently to infect previously Covid-positive and vaccinated people [11]. The National Institute for Communicable Diseases of South Africa reported that children under the age of 2 accounted for about $10 \%$ of total hospital admissions in Tshwane in South Africa [12]. The S gene target failure (SGTF) data of sampled cases in South Africa shows a growth rate of $21 \%$ per day compared to Delta, resulting in a 2.4-fold increase in the number of reproductions; Omicron became the majority strain in South Africa around 10 November. UK estimated an exceptionally high logarithmic growth rate of Omicron-associated SGTF cases over S gene target positive (SGTP) cases (to be 0.41 ) [10]. It was estimated to become the most dominant strain in the UK by December 14. Modeling of both genomic surveillance and SGTF data predicted that Omicron would become the most common variant nationally by December 25, 2021, with some areas surpassing this threshold earlier [13]. According to CDC, the cases due to Omicron variant may peak during early January 2022 [13]. Fig. 1 shows key timeline of Omicron variant and its prevalence rate in the United States.

\section{Mutation Profile of B.1.1.529}

The spike (S) protein, the principal antigenic target of antibodies produced by infections or immunization, has 32 mutations in this variant, which is exceptionally high. The deadly Delta variant, on the other hand, contains just $5 \mathrm{~S}$ protein mutations, posing a significant worldwide danger and spreading globally [14]. As a result, the panic button has been pressed in a number of cases throughout the world, and several nations have instituted travel restrictions to prevent the Omicron variant from spreading rapidly.

Omicron variant mutations are found in a variety of SARS-CoV-2 proteins, including S protein, envelope protein, membrane protein, NSP3, NSP4, NSP5, NSP6, NSP12, NSP14, and nucleocapsid protein [10]. The effect of changes in the $\mathrm{S}$ protein receptor-binding domain (RBD) on infectivity and antibody resistance is being investigated. This is because the RBD on the $\mathrm{S}$ protein facilitates in the $\mathrm{S}$ protein's attachment to the host angiotensin-converting enzyme 2 (ACE2). S-ACE2 binding facilitates SARS-CoV-2 entry into the host cell, which starts the viral infection process. There are at least 30 amino acid substitutions in the spike protein of the Omicron variant [13]. On top of that, there are three small deletions, and one small insertion. The RBD contains 15 of the 30 amino acid substitutions. Moreover, the variant has three mutations at the furin cleavage site which increases SARS-CoV-2 infectivity. Key amino acid substitutions in the spike protein are shown in Fig. 2 [13].

\begin{tabular}{|cccc}
\hline \multicolumn{4}{c}{ Spike } \\
\hline A67V & N856K & E484A & S371L \\
D614G & N969K & G339D & S373P \\
D796Y & P681H & G446S & S375F \\
L212I & Q954H & G496S & S477N \\
H655Y & T95I & K417N & T478K \\
L981F & T547K & N440K & Q493R \\
N679K & Y145D & N501Y & Q498R \\
N764K & del69-70 & Y505H \\
\multicolumn{4}{c}{ del142-144 } \\
del211 \\
ins214EPE
\end{tabular}

Fig. 2. Genomic structure of SARS-CoV-2 spike protein (top) [2] and the key mutations in the Omicron variant (bottom). The mutations in the red are in the RBD of the spike protein.

A total of 4 pango lineages are currently established to be associated with Omicron variant [15]. The most frequent sublineage is referred to as BA.1 (/B.1.1.529.1) while the two 
other sublineages are BA.2 (B.1.1.529.2) and BA.3 (B.1.1.529.3). Notably, BA.2 contrasts from the BA.1 by not containing the characteristic SGTF causing deletion $(\Delta 69-70)$ by which many PCR tests are able to detect a case as an Omicron, or Alpha, variant [10]. Due to this feature, BA.2 is also called the 'Stealth Omicron', however, both BA.2 and BA. 3 are very rare until now.

\section{Phylogenetics And Genome Evolution of}

\section{B.1.1.529}

According to ultrametric and metric clustering methods such as UPGMA and neighbor-joining, which used nucleotide substitution models that allowed the inclusion of nucleotide transitions and transversions as Kimura 80 models, the Omicron variant forms a new monophyletic clade that is distant from other SARS-CoV-2 variants, the Omicron variant forms a new monophyletic clade that is distant from other SARS-CoV-2 variants [16]. The neighbor-joining technique, on the other hand, revealed a close relationship between the Omicron variation and the recently produced Alpha variant when employing a simple nucleotide substitution model like Jukes-Cantor. Based on the percentage of sequence similarity, the closest variants were Omicron, Alpha, Gamma, Delta, Beta, Mu, and then the SARS-CoV-2 USA isolate. According to a genome alignment with other variants, the Omicron variant's genome contains the greatest gaps, ranging from 43 to 63 . Despite the fact that they evolved their own monophyletic group, Omicron is likely to have been there for considerably longer than predicted, given their close closeness to the Alpha variant [16]. To understand the virus's evolution and mutations, systematic and extensive sequencing activities are strongly advised.

\section{TRANSMisSion, BREAKTHROUGH INFECTION, AND VACCINE EFFICACY OF B.1.1.529}

The scientific community currently has little knowledge on infectivity, vaccine breakthrough, or antibody resistance of Omicron variant. Because the $\mathrm{S}$ protein, particularly its RBD, is so important in viral infection, vaccines and antibody medicines have focused on it. As a result, studying Omicron's 15 RBD mutations can help researchers learn more about the virus's infectivity, vaccine breakthrough, and antibody resistance. Several studies have revealed that the binding free energy (BFE) between the S RBD and the ACE2 is proportional to infectivity of virus [17], [18]. Consequently, a virus-killing antibody with a high affinity for the RBD could be developed [19], [20]. A substantial number of RBD binding antibodies are produced in the human immunological response to infection or vaccination. To treat viral infections, monoclonal antibodies (mABs) that target the $\mathrm{S}$ protein, namely the RBD, are utilized [19]. As a result, any alteration in the RBD of the $\mathrm{S}$ protein would raise urgent concerns about the efficacy of existing vaccinations, mAbs, and the danger of reinfection. A comprehensive experimental study of Omicron, on the other hand, may take weeks or even months. Chen et al. reported a quantitative investigation of infectivity, vaccination breakthrough, and antibody resistance of
Omicron variant in their article [21]. Omicron might be nearly ten times more contagious than the original virus or roughly twice as infectious as the Delta variant, according to an artificial intelligence (AI) model developed with tens of thousands of experimental data points and extensively confirmed by experimental data on SARS-CoV-2 [21]. It was discovered that Omicron may be twice as likely as Delta to evade existing vaccinations based on 132 3D models of RBDantibody complexes. They concluded that Eli Lilly's monoclonal antibodies (mAbs) that have been authorized by the Food and Drug Administration (FDA) may be gravely compromised and Omicron might potentially reduce the effectiveness of mAbs from Celltrion and Rockefeller University. However, very little effect on the Regeneron $\mathrm{mAb}$ combination has been found in their study.

In another study, Zhang et al. constructed the pseudotyped Omicron variant of SARS-CoV-2 [22]. The sensitivity of 28 serum samples from COVID-19 convalescent patients infected with the original SARS-CoV-2 strain was tested against pseudotyped Omicron, as well as other VOCs (Alpha, Beta, Gamma, and Delta) and VOIs (Lambda, Mu). The mean neutralization ED50 of these sera against Omicron declined to 66 (almost 8.4 times), as compared to the D614G reference strain $(\mathrm{ED} 50=556)$. The neutralization activity of other VOC and VOI pseudotyped viruses decreased just 1.2-4.5 times. According to the findings of their in vitro experiment, the Omicron version may cause a higher escape from immune protection generated by previous SARS-CoV-2 infection and maybe even current COVID-19 vaccines.

Collie et al. analyzed the effectiveness of BNT162b2 vaccine against Omicron Variant in South Africa [23]. For the comparator period, 133,437 PCR test results were obtained of which $28.6 \%$ had been obtained at least 14 days after the patient had received the second dose of vaccine. For the proxy omicron period, they analyzed 78,173 PCR test results, of which $41.4 \%$ were obtained at least 14 days after the second dose. During the comparator period, the overall test positivity was $6.4 \%$ while it was $24.4 \%$ during the proxy omicron period. Admission rate of Covid-19 was $10.8 \%$ and $2.2 \%$, respectively, as a percentage of positive PCR test results. During the proxy omicron period positive cases were more prevalent among younger population than during the comparator period. A vaccine efficacy of $70 \%$ (95\% CI: $62-$ $76 \%$ ) was found during the proxy omicron period, supported by the results of all sensitivity tests. On the other hand, the rate was 93\% (95\% CI: 90\%-94\%) against hospitalization for Covid-19 during the comparator period. Thus, the proxy omicron period maintains a reduced effectiveness of the BNT162b2 vaccine against hospital admission, this reduction is presumed to be due to the omicron variant.

In a recent study by $\mathrm{Ai}$ et al. Omicron variant showed lower neutralizing sensitivity than other SARS-CoV-2 variants to immune sera elicited by vaccines after boost [24]. The immunogenicity of COVID-19 breakthrough patients, the BBIBP-CorV homologous booster group, and the BBIBPCorV/ZF2001 heterologous booster group against SARSCoV-2 pseudotypes corresponding to the prototype, Beta, Delta, and the emerging Omicron variant was investigated. At 14 days following the homologous or heterologous vaccine boosters, 100 percent of samples demonstrated good neutralization activity against Omicron, despite a 
considerable drop (5.86-14.98 times) of pVNT against Omicron compared to the prototype. Their findings suggest that Omicron may be more prone to evade vaccine-induced immune protection than prototypes and other VOCs. Garcia Beltran et al. investigated neutralization potency of sera from 88 mRNA-1273, 111 BNT162b, and 40 Ad26.COV2.S vaccine recipients against wild type, Delta, and Omicron SARS-CoV-2 pseudo-viruses [25]. They considered three groups: recently vaccinated ( $<3$ months), distantly vaccinated (6-12 months), and recently boosted. Prior SARS-CoV-2 infection of these individuals was also considered. Omicron pseudovirus was more infectious than the other variants tested. Similar to the study of Ai et al., neutralization of Omicron was undetectable in most vaccinated individuals. Individuals boosted with mRNA vaccinations, on the other hand, demonstrated substantial neutralization of Omicron only 4-6-fold lower than wild type. This implies that booster doses increase the cross-reactivity of neutralizing antibody responses. In another investigation, neutralization titers against Omicron in serum samples from mRNA-1273 vaccination recipients were compared to neutralization titers against D614G and Beta in live- and pseudo-virus assays [26]. When blood samples were obtained 4 weeks following two standard inoculations with $100 \mathrm{~g}$ mRNA-1273, Omicron was 41-84 times less susceptible to neutralization than D614G and 5.3-7.4 times less responsive than Beta. The authors concluded that a booster dose increased Omicron neutralization titers and may substantially reduce the risk of symptomatic vaccine breakthrough infections, a conclusion consistent with the other studies [23]-[25].

\section{CONCLUSION}

With the development of Omicron, the fight against SARSCoV-2 has become more difficult. Due to its contagiousness and vaccine evading mutations, the newest SARS-CoV-2 variant Omicron (B.1.1.529) has sparked worldwide fear. A substantial number of mutations in the Spike protein imply that it may be changed in its responsiveness to immunological protection generated by the current COVID-19 infection and vaccinations. The World Health Organization's (WHO) designation of Omicron as a variation of concern (VOC) has prompted nations all over the world to impose travel restrictions and preventative measures. The SARS-CoV-2 variant's critical infectivity and antibody resistance are dictated by mutations in the $\mathrm{S}$ protein receptor-binding region. In this article, the current state of the art for a form of SARS-CoV-2 known as Omicron is reviewed, as well as a good foundation for future research initiatives was tried to build. As more data on these variations becomes accessible, more research is being done to better understand the polymorphisms and their impact on transmission, hospitalization, vaccination efficacy, and mortality. We expect that a thorough investigation of the Omicron variant will be conducted in order to minimize any unwanted implications that they and their mutations may have.

\section{REFERENCES}

[1] Chowdhury S, Bappy MH, Chowdhury S, Chowdhury MS, Chowdhury NS. COVID-19 induced cardiovascular complications and recent therapeutic advances. European Journal of Medical and Health Sciences. 2021; 3(6): 17-22.

[2] Chowdhury S, Bappy MH, Chowdhury S, Chowdhury MS, Chowdhury NS. Current Review of Delta Variant of SARS-CoV-2. European Journal of Medical and Health Sciences. 2021; 3(6): 23-9.

[3] Chowdhury S, Bappy MH. On the Delta Plus Variant of SARS-CoV2. European Journal of Medical and Health Sciences. 2021; 3(6): 525 .

[4] Mint. Omicron outbreak: List of countries where new Covid-19 variant has been detected. [Internet] 2021. [cited on November 27, 2021] Available from: https://www.livemint.com/news/world/omicronoutbreak-list-of-countries-where-new-covid-19-variant-has-beendetected-11638085299215.html

[5] World Health Organization Classification of Omicron (B.1.1.529): SARS-CoV-2 Variant of Concern. [Internet] 2021. [cited on November 27, 2021] https://www.who.int/news/item/26-11-2021-classificationof-omicron-(B.1.1.529)-sars-cov-2-variant-of-concern

[6] The New York Times. What we know about the Omicron variant.. [Internet] 2021. [cited on November 27, 2021] https://www.nytimes.com/2021/11/26/world/new-covid-variantomicron.html

[7] Mail \& Guardian. New Covid-19 variant is a concern as cases show a sharp increase in Gauteng. [Internet] 2021. [cited on November 27, 2021] https://mg.co.za/coronavirus-essentials/2021-11-25-new-covid19-variant-is-a-concern-as-cases-show-a-sharp-increase-in-gauteng/

[8] Wikipedia contributors. Timeline of the SARS-CoV-2 Omicron variant [Internet]. Wikipedia, The Free Encyclopedia. 2022. Available from: https://en.wikipedia.org/w/index.php?title=Timeline_of_the_SARSCoV-2_Omicron_variant\&oldid $=1063200523$

[9] CDC. COVID data tracker [Internet]. Centers for Disease Control and Prevention. 2020. [cited 2022 Jan 1]. Available from: https:/covid.cdc.gov/covid-data-tracker/

[10] Wikipedia contributors. SARS-CoV-2 Omicron variant [Internet]. Wikipedia, The Free Encyclopedia. 2022. Available from: https://en.wikipedia.org/w/index.php?title=SARS-CoV2 Omicron variant\&oldid $=1063146157$

[11] Pulliam JRC, van Schalkwyk C, Govender N, von Gottberg A, Cohen $\mathrm{C}$, Groome $\mathrm{MJ}$, et al. Increased risk of SARS-CoV-2 reinfection associated with emergence of the Omicron variant in South Africa [Internet]. bioRxiv. 2021 [cited 2022 Jan 1]. p. 2021.11.11.21266068. Available

from: https://www.medrxiv.org/content/10.1101/2021.11.11.21266068v2

[12] Bloomberg. COVID-19: Toddlers make up 10\% of hospital cases in South Africa's Omicron epicentre. Gulf News [Internet]. 2021. [cited 2022 Jan 1] Available from: https://gulfnews.com/world/africa/covid19-toddlers-make-up-10-of-hospital-cases-in-south-africas-omicronepicentre-1.84075071

[13] CDC. Science brief: Omicron (B.1.1.529) variant [Internet]. Centers for Disease Control and Prevention. 2021 [cited 2022 Jan 1]. Available from: https://www.cdc.gov/coronavirus/2019-ncov/science/sciencebriefs/scientific-brief-omicron-variant.html

[14] Chen J, Wang R, Gilby NB, Wei GW. Omicron (B.1.1.529): Infectivity, vaccine breakthrough, and antibody resistance. ArXiv [Preprint]. 2021.

[15] Outbreak.Info [Internet]. outbreak.info. [cited 2022 Jan 1]. Available from: https://outbreak.info/situationreports/omicron?loc $=$ ZAF\&loc $=$ GBR\&loc $=$ USA\&selected $=$ Worldwi de\&overlay $=$ false

[16] Kandeel M, Mohamed M, Abd El-Lateef HM, Venugopala KN, ElBeltagi HS. Omicron variant genome evolution and phylogenetics. Journal of Medical Virology. 2021.

[17] Wendong L, Zhengli S, Meng Y, Wuze R, Craig S, Jonathan EH, et al. Bats are natural reservoirs of SARS-like coronaviruses. Science. 2005; 310(5748): 676-679.

[18] Walls AC, Park Y-J, Tortorici MA, Wall A, McGuire AT, Veesler D. Structure, function, and antigenicity of the SARS-CoV-2 spike glycoprotein. Cell. 2020.

[19] Wang Chunyan, Li Wentao, Drabek Dubravka, Okba Nisreen MA, van Haperen Rien, Osterhaus Albert DME, van Kuppeveld Frank JM, Haagmans Bart L, Grosveld Frank, and Bosch Berend-Jan. A human monoclonal antibody blocking SARS-CoV-2 infection. Nature communications, 11(1):1-6, 2020.

[20] Li C, Tian X, Jia X, Wan J, Lu L, Jiang S, et al. The impact of receptorbinding domain natural mutations on antibody recognition of SARSCoV-2. Signal Transduction and Targeted Therapy. 2021; 6(1): 1-3.

[21] Chen J, Wang R, Gilby NB, Wei GW. Omicron (B.1.1.529): Infectivity, vaccine breakthrough, and antibody resistance. ArXiv [Preprint]. 2021. 
[22] Li Z, Qianqian L, Ziteng L, Tao L, Shuo L, Qianqian C, et al. The significant immune escape of pseudotyped SARS-CoV-2 Variant Omicron. Emerging Microbes \& Infections. 2021; 1-11.

[23] Collie S, Champion J, Moultrie H, Bekker L-G, Gray G. Effectiveness of BNT162b2 vaccine against omicron variant in South Africa. New Englands Journal of Medicine. 2021.

[24] Ai J, Zhang H, Zhang Y, Lin K, Zhang Y, Wu J, et al. Omicron variant showed lower neutralizing sensitivity than other SARS-CoV-2 variants to immune sera elicited by vaccines after boost. Emerging Microbes Infections. 2021; 1-24.

[25] Garcia-Beltran WF, St Denis KJ, Hoelzemer A, Lam EC, Nitido AD, Sheehan ML, et al. mRNA-based COVID-19 vaccine boosters induce neutralizing immunity against SARS-CoV-2 Omicron variant. medRxiv [Internet]. 2021. Available from: https://www.medrxiv.org/content/10.1101/2021.12.14.21267755v1.fu 11.pdf

[26] Doria-Rose NA, Shen X, Schmidt SD, O’Dell S, McDanal C, Feng W, et al. Booster of mRNA-1273 strengthens SARS-CoV-2 Omicron neutralization [Internet]. bioRxiv. 2021. Available from: http://dx.doi.org/10.1101/2021.12.15.21267805 